RAD Conference Proceedings, vol. 2, pp. 283-289, 2017

www.rad-proceedings.org

a.00001 tht

\title{
OPTICAL SPECIFICITY OF THIN SHELL FOR NANO-DELIVERY MODEL
}

\author{
Ana J. Šetrajčić-Tomić1 ${ }^{,}$Ljubiša D. Džambas², Jovan P. Šetrajčić $3^{*}$, \\ Matilda Vojnović4, Igor J. Šetrajčić3
}

1University of Novi Sad, Medical Faculty, Department of Pharmacy, Novi Sad, Vojvodina, Serbia
2University of Novi Sad, Medical Faculty, Department of Dentistry, Novi Sad, Vojvodina, Serbia
3University of Novi Sad, Faculty of Sciences, Department of Physics, Novi Sad, Vojvodina, Serbia

4The Health Center, Novi Sad, Vojvodina, Serbia

\begin{abstract}
The subject of the research in this paper includes theoretical investigation of nanomaterials modeling in the field of pharmaceutical technology for biomedical application. This includes a very precise encapsulated drug delivery, on exactly defined place in the human tissue or organ and a disintegration of capsule - drug carrier, so that the medicament can start producing its effect. The goal of multidisciplinary researches with biocompatible molecular nanomaterials is to find the parameters and the possibilities to construct boundary surfaces that will, in interaction with biological environment, create such properties of nanolayers that are convenient for use for layers of drug carrier capsules, biochips and biomarkers. These layers should demonstrate controlled disintegration of structure, better dielectric properties, discrete luminescence and appropriate bioporosity as all these are the requirements of contemporary nanomedicine. The main advantage of the theoretical approach is the essential knowledge of the mechanisms that allow us to comprehend the experimental conditions that we have to fulfill to be able to get the desired results. The results achieved up to now by our research group in the application of the Green's function method on flat ultrathin films are promising for applications in the frame of optical core-shell models. This paper presents the review of our current achievement in the field of theoretical physics of exciton ultrathin films and possible ways to materialize the same in the field of nanopharmacy.
\end{abstract}

Key words: Nanomedicine, optical biomaterials, ultrathin films, core-shell model, drug deliveries

DOI: $10.21175 / \operatorname{RadProc} .2017 .57$

\section{INTRODUCTION}

As we reported on last RAD conferences $[1,2]$ the development of biomedical science is directly related to the development of natural and mathematical sciences, especially materials science. Enormous resources are invested in the research that will lead to the solutions for many deadly diseases and in the end it is impossible not to put a completely logical question: whether the results of modern research justify the invested resources $[3,4]$ ?

Let us consider in what ways we can make contribution to research in the science. One approach is experimental, while the other is theoretical. There are big differences in the popularity of these approaches, yet both are said to have advantages and disadvantages too. Because we investigated the fundamental properties of low-dimensional structures, we will say something about this access: there are two aspects of the research approach. One uses computer resources, while the other uses analytic ones (literally speaking, paper and pencil). The aim of our research group is that within materials science we understand the essential mechanisms of transport processes in low-dimensional systems, so we chose an analytical approach with the ambition that in the very near future, we study with the help of computer resources too. Our method of choice is the Green's function which we use in the field of molecular crystals.

In this paper, we will present the continuation of our research related to the analysis of the application of nanomaterials in future nanopharmacy technology, i.e. in biomedicine, that is to say, we will present the recent accomplishments in basic and clinical nanomedicine. Numerous novel nanomedicine-related applications are under development or are in a research phase, and the process of converting basic research in nanomedicine into commercially viable products will be long and difficult. Achieving the full potential of nanomedicine may be years or even decades away, however, potential advances in drug delivery, diagnosis, and development of nanotechnology-related drugs are starting to change the landscape of medicine [1-4]. Site-specific targeted drug delivery (made possible by the availability of unique delivery platforms, such as dendrimers, nanoparticles and nanoliposomes) [5-7] and personalized medicine (result of the advance in pharmacogenetics) [8-10] are just a few concepts on the horizon of research.

*jovan.setrajcic@df.uns.ac.rs 


\section{DIELECTRICAL AND OPTICAL CHARACTERISTICS OF ULTRATHIN MOLECULAR CRYSTALLINE COATINGS}

The basic idea was to examine the exciton subsystem in bulk systems, and then in quasi-two dimensional ones, such as ultrathin coatings or films of molecular crystals, and then compare the results to get to micro-theory which would decipher the mechanisms of the optical properties of the observed system $[11,12]$.

Since we are interested in optical properties of molecular crystals, it is essential to observe the exciton subsystem. If we use the model of Frenkel's excitons with low concentrations, then the exciton subsystem in the harmonic approximation can be described by the following Hamiltonian [11-15]:

$$
H=\sum_{\vec{n}} \Delta_{\vec{n}} B_{\vec{n}}^{+} B_{\vec{n}}+\sum_{\vec{n}, \vec{m}} X_{\vec{n} \vec{m}} B_{\vec{n}}^{+} B_{\vec{m}}
$$

where $B_{n}^{+}$and $B_{\vec{n}}$ are creational and annihilation operators of an exciton at the node $\vec{n}$ of a crystal grid, $\Delta_{\vec{n}}$ is the energy of isolated exciton in that node, and $X_{\vec{n} \vec{m}}$ are matrix elements of excitons transfer from node $\vec{n}$ to node $\stackrel{i}{m}$. In the model it is assumed that the exciton energy at the node is $\sim 10^{2}$ times bigger than the energy of its transfer.

Our model of a crystal nanofilm assumes an ultrathin film confined by two parallel planes that are infinite in the $x$ and $y$ directions, while in the $z$ direction the film has a final thickness $L=N a$. We will observe a symmetrical nano-film (Fig. 1), which may be made by controlled reaching of massive specimens.

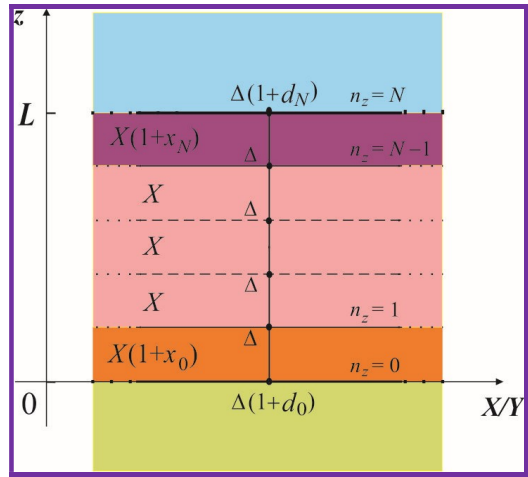

Figure 1. Ultrathin film mode

Due to the presence of boundary areas, the energies of excitons in the nodes and the energy transfers between boundary areas and neighboring areas are perturbed, where parameter $d$ defines perturbation at the node of boundary areas, and the parameter $x$ is a perturbation of transfer in boundary layers along the $z$ direction:

$$
\begin{gathered}
\Delta_{\vec{n}} \equiv \Delta\left(1+d_{0} \delta_{n_{z}, 0}+d_{N} \delta_{n_{z}, N}\right) ; \\
X_{\vec{n}, \vec{n}+\vec{\lambda}} \equiv X\left(1+x_{0} \delta_{n_{z}, 0}+x_{N} \delta_{n_{z}, N-1}\right) ; \\
X_{\vec{n}, \vec{n}-\vec{\lambda}} \equiv X\left(1+x_{0} \delta_{n_{z}, 1}+x_{N} \delta_{n_{z}, N}\right),
\end{gathered}
$$

Theoretical - quantum-mechanical analysis of the modeling system can be accomplished by solving the
Schrödinger's equation, or by Heisenberg's equations of motion with the use of "beyond DFT" methods based on $a b$ initio many body perturbation theory (MBPT) and involve solving the equations of motion of the one and two particle interacting Green's functions. Based on these methods, studies considering simulations emerged - using an appropriate software package [1618]. We opted for the tested and the adapted methods of the Green's function [11], using which the spectrum and the states of elementary excitations could be easily determined, as well as the equilibrium characteristics of the system [19]. The method of two-timed temperature Green's functions has been chosen for the microtheoretical analysis, due to its benefits - real parts of poles of Green's functions define energies of elementary excitations (from which the law of dispersion is obtained). If the Hamiltonian has a harmonic form, the Green's function has real poles only. The Green's function

$$
G_{\vec{n} \vec{m}}(t)=\left\langle\left\langle B_{\vec{n}}(t) \mid B_{\vec{m}}^{+}(0)\right\rangle\right\rangle,
$$

satisfies the certain the system of $N+1$ algebraicdifference equation of motion:

$$
\begin{aligned}
& G_{n_{z}, m_{z}}\left[\rho-\frac{\Delta}{|X|}\left(d_{0} \delta_{n_{z}, 0}+d_{N} \delta_{n_{z}, N}\right)\right]+ \\
& +G_{n_{z}+1, m_{z}}\left(1+x_{0} \delta_{n_{z}, 0}+x_{N} \delta_{n_{z}, N-1}\right)+ \\
& \quad+G_{n_{z}+1, m_{z}}\left(1+x_{0} \delta_{n_{z}, 1}+x_{N} \delta_{n_{z}, N}\right)=\frac{i \hbar}{2 \pi|X|} \delta_{n_{z}, m_{z}},
\end{aligned}
$$

where:

$$
\rho=\frac{\hbar \omega-\Delta}{|X|}+2\left(\cos a k_{x}+\cos a k_{y}\right),
$$

which define energy spectra of excitons in observed quantum model.

After the application of Green's functions [11-15] one can obtain the expression which represents the relative dynamical permittivity as a function of the frequency of initial electromagnetic excitation:

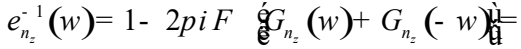

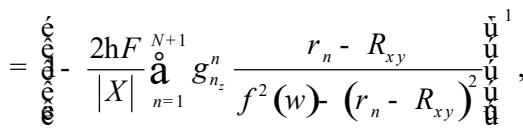

where are: $F-$ is structural factor [19], $f(\omega)=\hbar \omega /|X|$ and $R_{x y}=\Delta /|X|-2\left(\cos a k_{x}+\cos a k_{y}\right)$.

Our scope of research is optic properties of the observed model example. The refraction $(n)$ and absorption $(\kappa)$ indices are usually defined in the literature [13-15] by permittivity term: $\sqrt{\varepsilon}=n+i \kappa$. Introducing the complex frequency: $\omega=\omega+i v$ in expression for permittivity (6), we get complex permittivity: $\varepsilon=\varepsilon^{\prime}+i \varepsilon^{\prime \prime}$. Based on this, and bearing in mind that permittivity depends on the position in the film: $\varepsilon=\varepsilon\left(n_{z}\right)$, we can find the expression for absorption and refraction indices in the following form: 


$$
\begin{aligned}
& \kappa_{n_{z}}(\omega)=\sqrt{\frac{\varepsilon_{n_{z}}^{\prime}(\omega)}{2}\left\{\sqrt{1+\left[\frac{\varepsilon_{n_{z}}^{\prime \prime}(\omega)}{\varepsilon_{n_{z}}^{\prime}(\omega)}\right]^{2}}-1\right\}} ; \\
& n_{n_{z}}(\omega)=\sqrt{\frac{\varepsilon_{n_{z}}^{\prime}(\omega)}{2}\left\{\sqrt{1+\left[\frac{\varepsilon_{n_{z}}^{\prime \prime}(\omega)}{\varepsilon_{n_{z}}^{\prime}(\omega)}\right]^{2}}+1\right\}},
\end{aligned}
$$

The reflection $(r)$ and transparency $(t)$ indices are defined $\left[13^{-15}\right]$ by the refraction and absorption ones:

$$
\begin{aligned}
& r_{n_{z}}(\omega)=\frac{\left[n_{n_{z}}(\omega)-1\right]^{2}+\kappa_{n_{z}}^{2}(\omega)}{\left[n_{n_{z}}(\omega)+1\right]^{2}+\kappa_{n_{z}}^{2}(\omega)} ; \\
& t_{n_{z}}(\omega)=1-n_{n_{z}}(\omega)-\kappa_{n_{z}}(\omega) .
\end{aligned}
$$

From this and the expressions, one can see that dielectric and all optical characteristics of the film depend on the position of film-layer.

By numerical calculations, we have analyzed dynamical permittivity and optical indices behavior on plane position and on values of boundary parameters, and calculated that. The number of resonant peaks directly depends on the film width, i.e., on the number of layers $N$ (there $N=3$ ). General rule is that the number of resonance peaks decreases inside layers of the film, although with the influence of perturbation parameters $d_{0, N} \in[-0.2,+0.2]$ and $x_{0, N} \in[-0.5,+1.0]$ that rule can be broken, and in that case, (maximal) number of peaks is $N+1 \equiv 4$, with the possibility of some resonance peaks disappear, i.e. they overlap or vanish.

Subsequently, we continued with the research of the same structures and investigated the influence of boundary parameters on optical properties of these structures, for different values of perturbation parameters. Graphics on Fig. 2 shows some results for representative cases for three/layered asymmetrically perturbed film, with: $d_{0}=-0.1, d_{N}=0.1 ; x_{0}=-0.5$ and $x_{N}=1.0$.

Relative dynamic permittivity of bulk samples of molecular crystals have a complete absorption zone in infrared (IR) area [9], corresponding to absorption of all IR rays with relative frequency $f \in(43.5,47.5)$. This means that the optical indices of the bulk sample, in this frequency interval, having the following values: $\kappa \mathrm{b}$ $=1, r_{\mathrm{b}}=\tau_{\mathrm{b}}=0$ and $n_{\mathrm{b}}=n$. On the contrary, in ultrathin films only discrete absorption lines will occur, and their number corresponds to the number of possible exciton states. Distribution and intensity of absorption lines depend on the value of the boundary parameters!

From the presented graphics one can see that the film-structures have discrete and selective optical properties, there are maxima 4 discrete absorption lines, equal to number of possible (macro) quantum states of excitons through film-thickness $[10,15]$.

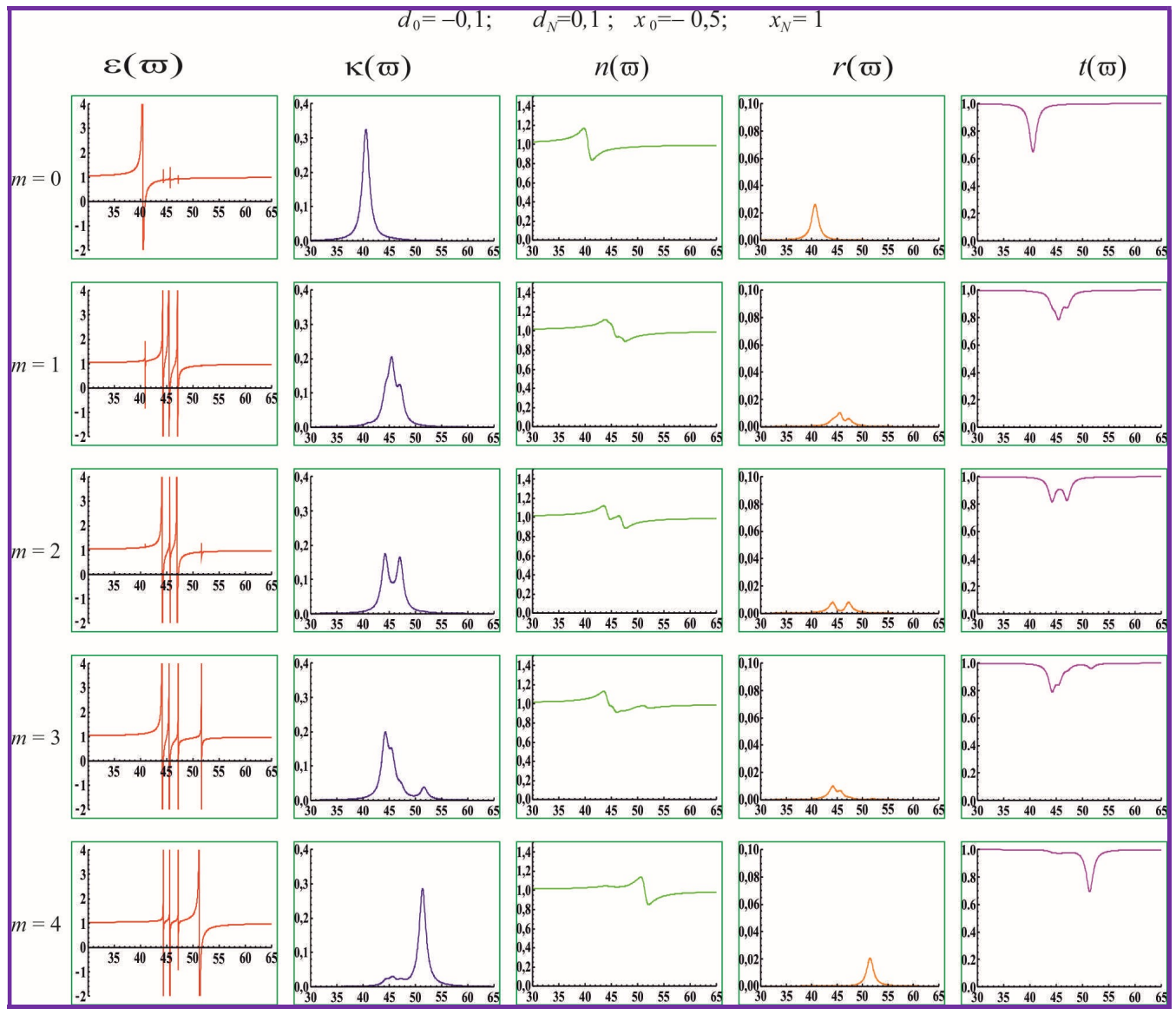

Figure 2. Relative permittivity and relevant optical indices of perturbed four-layered film 
Since the emission and absorption spectra can be experimentally recorded but only for the whole film, we have determined these optical characteristics (indices of absorption and refraction) not only for the particular layers but for the whole shell as well and in the normal direction on boundary surface of the shell (or radial from core center) where the changes of these values occur in relation to their bulk values.

Because the index of absorption is part of the energy/intensity electromagnetic radiation which is absorbed by the film in relation to the total incident energy/intensity of the radiation that got to the film, it can be concluded that the overall indices of absorption $\left(\kappa_{F}\right)$, reflection $\left(r_{F}\right)$ and transparency $\left(t_{F}\right)$ for the whole film could be acquired by simply adding up adequate indexes on individual planes, i.e.

$$
\phi_{F}(\omega)=\sum_{n_{z}} \phi_{n_{z}}(\omega) ; \quad \phi \equiv(\kappa, r, \tau) .
$$

Of course, this summation means that the addition is done strictly by the individual, i.e., specific frequencies, because it is a question of values of relative dynamic quantities.

In contrast, refractive indexes present the ratios of appropriate speeds, and therefore additivity and simple sum cannot be applied to them. It is not difficult to show $[13-15,20]$, that the refractive index for whole film can be calculated as a sum of reciprocal values; the rule of the sum of reciprocal values must be applied in the following form:

$$
\frac{N}{n_{F}(\omega)}=\sum_{n_{z}} \frac{1}{n_{n_{z}}(\omega)} .
$$

Based on the last two formulas, we numerically determined the dependence of the absorption index and the refraction index for the whole film. The results of these calculations are presented in Fig. 3.

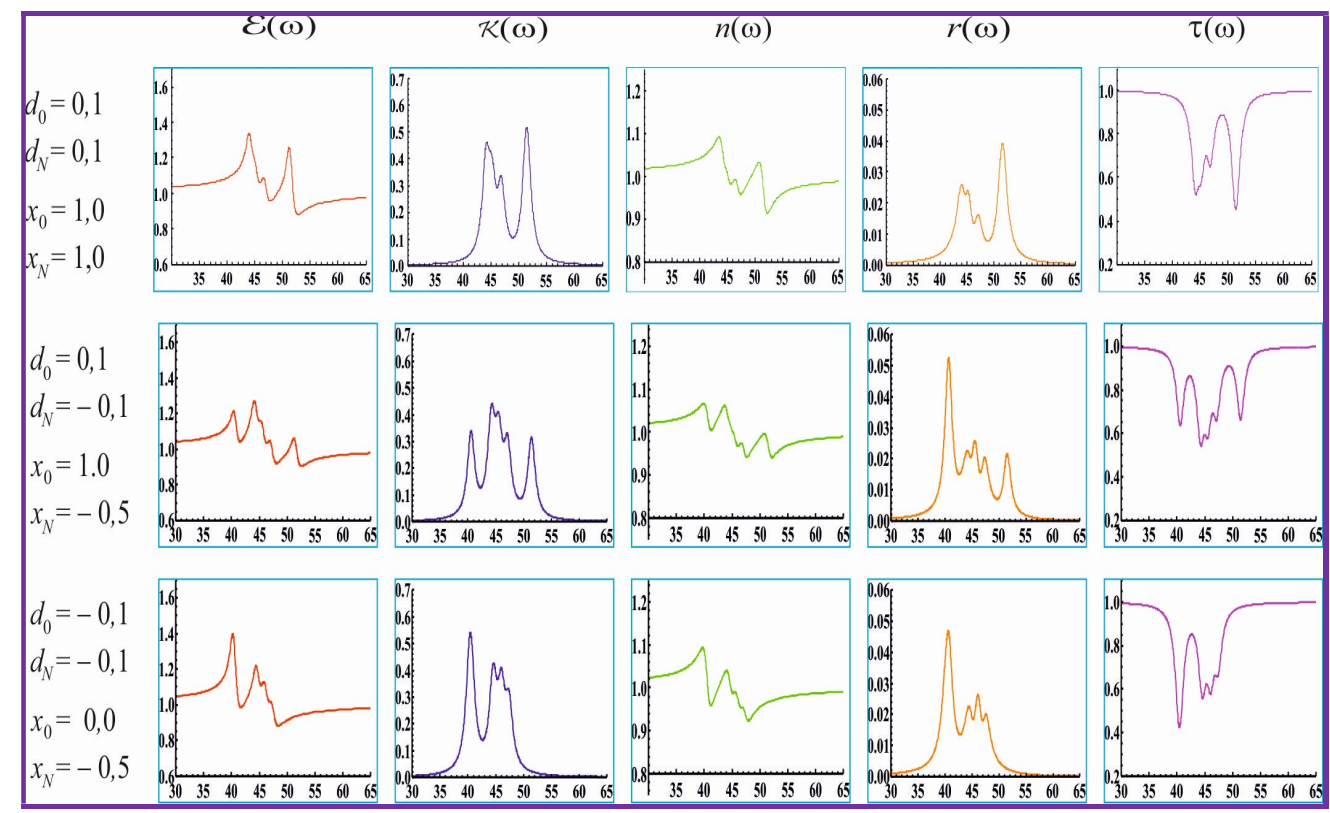

Figure 3. Relative permittivity and relevant optical indices of perturbed four-layered film

By numerical calculations, we have analyzed the dynamical permittivity and optical indices behavior on plane position and on values of boundary parameters, and calculated those values. The number of resonant peaks directly depends on the film width, i.e., on the number of layers $N$ (there $N=4$ ). General rule is that the number of resonance peaks decreases inside layers of the film, although with influence of perturbation parameters $d_{0, N} \in[-0.2,+0.2]$ and $x_{0, N} \in[-0.5,+1.0]$ that rule can be broken and in that case (maximal) number of peaks is $N+1 \equiv 5$, with possibility of some resonance peaks to disappear, i.e. they overlap or vanish.

Subsequently, we continued with the research of the same structures and investigated the influence of boundary parameters on optical properties of these structures, for different values of perturbation parameters. From the presented graphics one can see that the film-structures have discrete and selective optical properties, there are maxima 5 discrete absorption lines, equal to the number of possible (macro) quantum states of excitons through filmthickness [10,15].

For the case of bulk there is a completely clear absorption zone, while for the case of films there are $\leq$ 5 absorption peaks corresponding to the resonant lines.

Refraction indices have jumps on the places where the absorption index changes: for the bulk, that is happening on the borders of the absorption zone, while the film has $1-5$ corresponding, not very narrow peaks. Reflection for the bulk is only out of the borders of the absorption zone, the reflection for films is partial and it happens on each crystallographic plane.

The most interesting result is definitely nontransparency of molecular bulk, but when it is thinned to the film of nano-size it becomes transparent, except 
for maxima 4 discrete peaks which correspond to the combination of absorption and reflection peaks of the film.

\section{Potential RESEARCH OF CORE ULTRATHIN MULTI- SHELL NANODELIVERY MODELS}

As reported earlier [1,2], our research group believes that the methodology used to analyze optical properties of nano-films can be used for theoretical studies of materials that are typical for drug carriers in nano-pharmacy. We are primarily thinking of core shell models under which the systems include a spherical shape. The basic idea of core shell model in biomedicine is to fill the interior of hollow nanospheres by active component of pharmaceuticals. The role of hollow spheres measuring up to tens of nanometers is transport. Thanks to its dimensions and properties, hollow nano-spheres are ideal candidates to be hosts for the active components of pharmaceuticals, which cannot be done by conventional methods without serious consequences transported to the target place. Take the example of ketoprofen, a good conventional nonsteroidal anti-inflammatory agent; oral intake can lead to ulcer. Second, but unconventional way of entering the body is through polymeric nano-particles such as PDLLA (poly-DL-lactide acid). The result is the introduction of pharmaceuticals as a cream, which would be rubbed in determined places that will transfer it, rather than orally.

The main advantage of the theoretical approach is the essential knowledge of the mechanisms that allow us to comprehend the experimental conditions that must be fulfilled to get the desired results. The results achieved up to now by our research group in the application of the Green's function method on flat ultrathin films are promising for applications in the frame of core-shell models. This paper presents the review of our current achievement in the field of theoretical physics of ultrathin films and the possible ways to materialize the same in the field of nanopharmacy.

It is certain that a solution to the problems of delivery and transport of pharmaceuticals in different parts of the body lies in the achievements of materials science. In this sense, nano-materials and hollow nanospheres are materials which will drive attention in the future even more than now. For positive results in this area, many more experimental methods are used. Even those theoretical mainly represent the results obtained using the computing resources, as opposed to analytical methods [20-27].

Our goal is the use and adaptation of analytical methodology applied to flat ultrathin films on the spherical systems that look as if composed of a certain number of ultrathin films [28-30].

\section{CONCLUSION}

Our model of a crystal nanofilm assumes an ultrathin film confined by two parallel planes that are infinite in the $x$ and $y$ directions, while in the $z$ direction the film has a final thickness $L=N a$. We will observe a symmetrical nano-film (Fig. 1), which may be made by controlled reaching of massive specimens.
Due to the presence of boundary areas, the energies of excitons in the nodes and the energy transfers between boundary areas and neighboring areas are perturbed, where parameter $d$ defines perturbation at the node of boundary areas, and the parameter $x$ is a perturbation of transfer in boundary layers along the $z$-direction.

It is certain that a solution to the problems of delivery and transport of pharmaceuticals in different parts of the body lies in the achievements of materials science. In this sense, nano-materials and hollow nanospheres are the materials which will get attention in the future even more than now. For positive results in this area, many more experimental methods are used. Even those theoretical mainly represent the results obtained using the computing resources, as opposed to analytical methods.

Our goal is the use and adaptation of analytical methodology applied to flat ultrathin films on the spherical systems that look as if composed of a certain number of ultrathin films.

The basic idea was to examine exciton subsystem in the bulk systems, and then in quasi-two dimensional, such as ultrathin coatings or films of molecular crystals, and then compare the results to get to microtheory which would decipher the mechanisms of the optical properties of the observed system.

The most important result is definitely the nontransparency of molecular bulk, but when it is thinned to the film of nano-size it becomes transparent except for maxima 4 discrete peaks which correspond to the combination of absorption and reflection peaks of the film.

Acknowledgement: The paper is a part of the research done within the projects ON-171039 and TR-34O19 (Ministry of Education, Sciences and Technological Development of the Republic of Serbia), and the project 19/6-020/961-16/15 (Ministry of Science and Technology of the Republic of Srpska) as well as the project 114--451--2092 (Provincial Secretariat for Science and Technological Development of Vojvodina).

\section{REFERENCES}

1. J. P. Šetrajčić, A. J. Šetrajčić-Tomić, Lj. D. Džambas and I. Gušić, "Core-shell layered models of nanostructured carriers for a nano-bio-medical applications," in Proc. of 3rd International Conference on Radiation and Applications in Various Fields of Research, Budva, Montenegro, 2015, pp. 487-494.

Retrieved from:

http://www.radconference.org/helper/download.php?file=../pdf/Proceed ings\%20RAD\%202015.pdf

Retrieved on: Feb. 8, 2016

2. A. J. Šetrajčić-Tomić, Lj. D. Džambas, D. Lj. Džambas and J. P. Šetrajčić, "Optical Core-Shell Model for NanoDelivery Applications," in Proc. of 4th International Conference on Radiation and Applications in Various Fields of Research, Niš, Serbia, 2016, pp. 165-170.

DOI: 10.21175/RadProc.2016.39

3. K. J. Morrow Jr., R. Bawa, C. Wei, "Recent advances in basic and clinical nanomedicine," Med. Clin. N. Am, vol. 91, no. 5, pp. 805-843, Sep. 2007.

DOI: 10.1016/j.mcna.2007.05.009

PMid: 17826104

4. Drug Delivery Nanoparticles: Formulation and Characterization, vol. 191, Y. Pathak, D. Thassu, Eds., 1st 
ed., New York (NY), USA: Informa Healthcare USA Inc., 2009.

Retrieved from:

http://file.zums.ac.ir/ebook/231-

Drug\%20Delivery\%20Nanoparticles\%20Formulation\%2 oand\%20Characterization,\%20Vol.\%202-

Yashwant\%20Pathak\%20\%20Deepak\%20.pdf Retrieved on: Jan. 16, 2017.

5. H. E. Schaefer, Nanoscience - The science of the small in physics, engineering, chemistry, biology and medicine, vol. 1, 1st ed., Berlin, Germany: Springer, 2010.

DOI: $10.1007 / 978-3-642-10559-3$

6. M. A. Greenwood, "Are Quantum Dots on the Brink of Their Big Break?" Photonic Spectra, vol. 41, no. 5, May 2007.

Retrieved from:

www.photonics.com/Article.aspx?AID=29421

Retrieved on: Jan. 14, 2017.

7. E. A. Murphy, B. K. Majeti, L. A. Barnes, M. Makale, S. M. Weis, K. Lutu-Fuga, W. Wrasidlo, D. A. Cheresh, "Nanoparticle-Mediated Drug Delivery to Tumor Vasculature Suppresses Metastasis," Proc. Natl. Acad. Sci. USA, vol. 105, no. 27, pp. 9343-9348, July 2008.

DOI: $10.1073 /$ pnas.0803728105

PMid: 18607000

PMCid:PMC2453735

8. W. C. W. Chan, "Bionanotechnology Progress and Advances," Biol. Blood Marrow Transplant, vol. 12, no. 1, pp. 87-91, Jan. 2006.

DOI: 10.1016/j.bbmt.2005.10.004

PMid: 16399591

9. Y. Pathak, "Recent Developments in Nanoparticulate Drug Delivery Systems," in Drug Delivery Nanoparticles: Formulation and Characterization, Y.Pathak, D.Thassu, Eds., 1st ed., New York (NY), USA: Informa Healthcare USA Inc., 2009, ch. 1, pp.1-15.

Retrieved from:

http://file.zums.ac.ir/ebook/231Drug\%20Delivery\%20Nanoparticles\%20Formulation\%2 oand\%20Characterization,\%20Vol.\%202-

Yashwant\%20Pathak\%20\%20Deepak\%20.pdf Retrieved on: Jan. 16, 2017

10. H. Devapally, A. Chakilam, M. M. Amiji, "Role of Nanotechnology in Pharmaceutical Product Development," J. Pharm. Sci., vol. 96, no. 10, pp. 25472565, Oct. 2007.

DOI: 10.1002/jps.20875

PMid: 17688284

11. J.P. Šetrajčić, "Exact Microtheoretical Approach to Calculation of Optical Properties of Ultralow Dimensional Crystals," arXiv: 1004.2387 [cond-mat.mtrl-sci], April 2010.

Retrieved from:

https://arxiv.org/abs/1004.2387

Retrieved on: Jan. 12, 2017

12. J. P. Šetrajčić, S. S. Pelemiš, S. M. Vučenović, V. M. Zorić, S. Armaković, B. Škipina, A. J. Šetrajčić, "Absorption Features of Symmetric Molecular Nanofilms," in IEEE Proceedings 27th International Conference on Microelectronics, Niš, Serbia, 2010, vol. 1, pp. 127-130. Retrieved from:

http://ieeexplore.ieee.org/xpl/mostRecentIssue.jsp?punu mber $=5483038$.

Retrieved on: Jan. 12, 2017.

13. B. Škipina, D. Lj. Mirjanić, S. M. Vučenović, J. P. Šetrajčić, I. J. Šetrajčić, A. J. Šetrajčić-Tomić, S. S. Pelemiš, B. Markoski, "Selective IR Absorption in Molecular Nanofilms," Optical Materials, vol. 33, no. 11, pp. 1578-1584, Sep. 2011. DOI: 10.1016/j.optmat.2011.04.008

14. I. J. Šetrajčić, D. Rodić, J. P. Šetrajčić, "Optical Properties of Layers of Symmetric Molecular Nanofilms," J. Opt., vol. 44, no. 1, pp. 1-6, Mar. 2015. DOI: $10.1007 / \mathrm{s} 12596-014-0231-8$
15. J. P. Šetrajčić, D. Rodić, V. D. Sajfert, N. Pop, M. D. Popov, "Confinement Consequences on Optics of Molecular Crystalline Nanofilms," Quantum Matter, vol. 6, no. 1, pp. 1-3, Feb. 2017 DOI: $10.1166 / \mathrm{qm} .2017 .1392$.

16. Su Y. Quek, J. B. Neaton, M. S. Hybertsen, E. Kaxiras, S. G. Louie, "First-principles Studies of the Electronic Structure of Cyclopentene on Si(001): Density Functional Theory and GW Calculations," Phys. Stat. Solidi (b), vol. 243, no. 9, pp. 2048-2053, Jul. 2006. DOI: $10.1002 / \mathrm{pssb} .200666819$

17. G. Samsonidze, M. Jain, J. Deslippe, M. L. Cohen S. G. Louie, "Simple Approximate Physical Orbitals for GW Quasiparticle Calculations," Phys. Rev. Lett., vol. 107, no. 18, p. 186404, Oct. 2011.

DOI: 10.1103/PhysRevLett.107.186404 PMid: 22107653

18. J. Deslippe, G. Samsonidze, D. A. Strubbe, M. Jain, M. L. Cohen, S. G. Louie, "BerkeleyGW: A Massively Parallel Computer Package for the Calculation of the Quasiparticle and Optical Properties of Materials and Nanostructures," Comput. Phys. Commun., vol. 183, no. 6, pp. 1269-1289, Jun. 2012. DOI: 10.1016/j.cpc.2011.12.006

19. G. Mahan, Many Particle Physics, 3rd ed., New York (NY), USA: Springer, 2000. DOI: $10.1007 / 978-1-4757-5714-9$

20. J. P. Šetrajčić, D. Lj. Mirjanić, S. M. Vučenović, D. I. Ilić, B. Markoski, S. K. Jaćimovski, V. D. Sajfert, V. M. Zorić, "Phonon Contribution in Thermodynamics of NanoCrystalline Films and Wires," Acta Phys. Pol. A, vol. 115, no. 4, pp. 778-782, Apr. 2009.

DOI: 10.12693/APhysPolA.115.778

21. S. M. Stojković, D. Lj. Mirjanić, J. P. Šetrajčić, D. D. Šijačić, I. K. Junger, "Spectra and States of Electrons in Surface Perturbed Quantum Wires," Surface Science, vol. 477, no. 2-3, pp. 235-242, April 2001. DOI: 10.1016/So039-6028(01)00887-1

22. D. I. Ilić, S. M. Vučenović, S. K. Jaćimovski, V. M. Zorić, J. P. Šetrajčić, "Phonon Spectra and Thermodynamics of Crystalline Nanowires, in Low-Dimensional Materials Synthesis, Assembly, Property Scaling, and Modeling," Mater. Res. Soc. Proc., vol. 1017, pp. 1-6, Jan. 2007. DOI: 10.1557/PROC-1017-DDo8-50

23. I. D. Vragović, J. P. Šetrajčić, R. Scholz, "Quantum Size Effects in the Optical Properties of Organic Superlattices Containing 3,4,9,10 PTCDA," Eur. Phys. J. B, vol. 66, no. 2, pp. 185-190, Nov. 2008. DOI: $10.1140 /$ epjb/e2008-00409-1

24. J. P. Šetrajčić, S. K. Jaćimovski, "Review of Results of Theoretical Approaches to Phonon Engineering of Thermodynamic Properties for Different Quantum Structures," $N B P$, vol. 20, no. 3, pp. 67-82, 2015 DOI: 10.5937/NBP1503067J

25. J. P. Šetrajčić, "Research of Properties of Nanoscopic Structures," Zaštita materijala, vol. 57, no. 1, pp. 81-92, 2016.

Retrieved from:

http://www.readcube.com/articles/10.5937/ZasMat1601 o81S Retrieved on: Jan. 13, 2017.

26. J. P. Šetrajčić, V. D. Sajfert, S. K. Jaćimovski, "Fundamental Preferences of the Phonon Engineering for Nanostructural Samples,” Rev. Theor. Sci., vol. 4, no. 4 pp. 353-401, Dec. 2016. DOI: $10.1166 /$ rits.2016.1067

27. G. Samsonidze, F. J. Ribeiro, M. L. Cohen, S. G. Louie, "Quasiparticle and Optical Properties of PolythiopheneDerived Polymers," Phys. Rev. B, vol. 9o, no. 3, p. 035123 , Jul. 2014 DOI: 10.1103/PhysRevB.90.035123

28. D. Lj. Mirjanić, J. P. Šetrajčić, Lj. D. Džambas, V. D. Mirjanić, A. J. Šetrajčić - Tomić, V. M. Zorić "Nanoscopic Biomaterials in Medicine, Dentistry and 
Pharmacy," presented at the 1oth International Scientific-Practical Conference: Research, Development and Application of High Technologies in Industry, Saint-Petersburg, Russia, 2010.

29. J. P. Šetrajčić, D. Lj. Mirjanić, A. J. Šetrajčić-Tomić, S. Armaković, I. J. Šetrajčić, "Core-Shell Nanomodels for Biomedical Applications," in Proc. International Conference - New face of TMCR: Modern Technologies, Quality and Innovation, Chisinau, Moldova, 2011, pp. 545-548.
30. J.P. Šetrajčić, D. Rodić, A. J. Šetrajčić-Tomić S. M. Vučenović, "Resonant Optical Occurrences in Molecular Nanofilms", Proc. 8th Internationl Conference: Contemporary Materials, Banja Luka, Rep. Srpska, 2016, pp. 71-78. 